\title{
Autoimmunity in Common Variable Immunodeficiency
}

\author{
Shradha Agarwal, MD and Charlotte Cunningham-Rundles, MD, PhD
}

\section{Abstract}

Common variable immunodeficiency (CVID) is the most common clinically significant primary immune defect. Although the hallmark of CVID is hypogammaglobulinemia, the intrinsic dysregulation of the immune system leads to defective T-cell activation and proliferation, as well as dendritic cell and cytokine defects. Although $70 \%$ to $80 \%$ of patients have had recurrent sinopulmonary infections, auto-immunity and inflammatory complications are also common. The most common autoimmune conditions are immune thrombocytopenic purpura and hemolytic anemia, but other autoimmune complications arise, including rheumatoid arthritis, pernicious anemia, primary biliary cirrhosis, thyroiditis, sicca syndrome, systemic lupus, and inflammatory bowel disease. Treatment of autoimmunity includes high-dose immunoglobulins, corticosteroids, selected immunosuppressants, and other immune modulators. This review focuses on autoimmune conditions associated with CVID, potential mechanisms of immune dysregulation, and therapeutic strategies.

\section{Introduction}

The diagnosis of common variable immunodeficiency (CVID) is based on significantly reduced levels of IgG and IgA and/or IgM compared with age-related standards, accompanied by impaired or absent antibody production [1]. CVID is essentially a diagnosis of exclusion, as other causes of hypogammaglobulinemia, including known gene defects, medications, protein loss, or malignancy, must be excluded. Unlike other genetic immune defects, CVID is generally diagnosed in the third or fourth decade of life; however, a diagnostic delay of 6 to 8 years after the appearance of the first characteristic symptom is common $[2 \bullet \bullet, 3]$. About $70 \%$ to $80 \%$ of patients have recurrent sinopulmonary infections, but others have additional medical conditions, including gastrointestinal infectious or inflammatory disease, lymphadenopathy, splenomegaly, and/or autoimmune diseases. The pathogenesis of autoimmunity in CVID remains obscure. How autoantibodies can be produced against specific tissues in a state of impaired antibody production is unclear. Harder to understand is the pathogenesis of lymphoid hyperplasia, granulomatous infiltrations, and inflammatory bowel disease, as these are more inflammatory syndromes than strictly autoimmune-based conditions. This review outlines the autoimmune conditions in CVID and current treatment options and highlights what is known about the genetics and immune dysregulation in this syndrome.

\section{Autoimmune Conditions}

Autoimmune diseases affect about $20 \%$ of CVID patients and are commonly the first manifestation of immune deficiency (Table 1) $[2 \bullet \bullet, 3]$. In one study, autoimmunity was

Corresponding author: Charlotte Cunningham-Rundles, MD, PhD, Mount Sinai Medical Center, 1425 Madison Avenue, New York, NY 10029, USA., charlotte.cunningham-rundles@mssm.edu.

Disclosure

No potential conflicts of interest relevant to this article were reported. 
found before the diagnosis of CVID in $17.4 \%$ of 224 patients, and in $2.3 \%$ of the $17.4 \%$ of these patients, autoimmune disease was the only clinical manifestation at the time of diagnosis of CVID [4]. Although males and females appear to have autoimmunity in equal numbers, data from the recent European Society for Immune Deficiency CVID registry noted a higher prevalence of autoimmunity in the United Kingdom compared with Sweden, suggesting differences in patient referrals, environmental factors, or possibly genetic backgrounds $[2 \cdot \bullet]$.

\section{Hematologic autoimmunity and lymphoid hyperplasia}

The most common conditions are immune thrombocytopenic purpura (ITP) and autoimmune hemolytic anemia (AIHA), which occur singly or consecutively or concurrently with Evans syndrome. The prevalence of ITP/AIHA is estimated to be approximately $5 \%$ to $8 \%$ in patients with CVID [5]. In one study of 326 CVID patients, the prevalence of hematologic cytopenias was $11 \%(n=35)$ : 15 with ITP, 9 with AIHA, and 11 with Evans syndrome. Most of these patients ( 30 of 35) developed autoimmune disease before or concurrent with the diagnosis of CVID and start of immunoglobulin (Ig) therapy [6]. Autoimmune neutropenia appears to be rarer than ITP or AIHA.

With or without a history of ITP or AIHA, an enlarged spleen is common in CVID. In one study of 224 CVID patients, splenic enlargement (longitudinal diameter $>11 \mathrm{~cm}$ ) was noted in $17.3 \%$ of patients at the time of diagnosis [4]. At the last follow-up (mean, 11.5 years), the prevalence of splenomegaly increased to $26.4 \%$. Splenomegaly was usually associated with liver enlargement and/or abdominal lymphadenopathy. In $6 \%$ of these patients, the histology of lymph nodes, liver, and spleen involved a granulomatous reaction [4]. In the European Society for Immune Deficiency CVID registry, polyclonal lymphocytic infiltration (defined as lymphoid interstitial pneumonitis, unexplained granuloma, unexplained hepatomegaly, splenomegaly, and extensive and persistent lymphadenopathy) was correlated with the serum IgM level. For each additional $1 \mathrm{~g} / \mathrm{L}$ of $\operatorname{IgM}$, there was a $16 \%$ increased odds that the patient would develop polyclonal lymphocytic infiltration and a $31 \%$ increased odds that the patient would have a lymphoid malignancy [2••].

Treatment of immune cytopenias with oral or intravenous corticosteroids and/or high-dose intravenous immunoglobulin $(1 \mathrm{~g} / \mathrm{kg})$ generally is successful, although anti-rhesus D Ig or, more recently, the anti-CD20 monoclonal antibody rituximab may be required [6,7]. In many cases, ITP and/or AIHA is accompanied by an enlarged spleen and lymphadenopathy elsewhere. However, splenectomy in patients with CVID should be avoided given the susceptibility to infection with encapsulated organisms. In two series, splenectomy led to complications: $10 \%$ of patients had pneumococcal sepsis and $16 \%$ had postoperative infections [5]. Replacement Ig may reduce the reoccurrence of autoimmunity in CVID, as most CVID patients studied in one group had one or more episodes of hematologic autoimmunity before or at the time of diagnosis of CVID, whereas only a small minority had either condition after Ig was initiated [6].

\section{Rheumatologic diseases}

Joint manifestations resembling rheumatoid arthritis or juvenile rheumatoid arthritis occur in $1 \%$ to $10 \%$ of patients with CVID $[5,8,9]$. These generally include symmetric involvement of few or many joints—commonly the knees, ankles, and hands—and may result in joint destruction. Antinuclear antibodies or a rheumatoid factor are typically absent due to lack of antibody production in CVID. Histologic abnormalities of the synovial membrane are unlike those seen in patients with typical rheumatoid arthritis. There may be synovial hyperplasia and capillary proliferation without major lymphocytic or polymorphonuclear infiltrate, but few to no B cells and no plasma cells, and T-cell infiltrate may be composed of CD8 ${ }^{+}$cells. 
Clinically, monoarticular arthritis in CVID is more commonly a result of infections such as Streptococcus pneumoniae, Haemophilus influenzae, or Staphylococcus aureus. However, infections with atypical organisms such as Mycoplasma hominis, Mycoplasma pneumoniae, Mycoplasma salivarium, and Ureaplasma urealyticum are harder to diagnosis and may necessitate a trial of an antibiotic to exclude such an infection [8,9].

Systemic lupus erythematosus (SLE) appears to be uncommon in CVID but has been described. In a review of 18 patients with CVID and SLE, 89\% were females (mean age at onset of SLE, 23.8 years) [10]. The most frequent symptom in these patients was a sinopulmonary infection; $60 \%$ had reduced numbers or percentages of B cells. Fifty percent of patients developed CVID within 5 years of the diagnosis of SLE. Curiously, in 67\%, the SLE disease activity decreased after the development of CVID, suggesting that loss of B cells or functional antibody may have contributed to clinical remission [10]. However, other studies have reported patients with exacerbations of SLE disease activity after development of hypogammaglobulinemia, suggesting that more than one mechanism may be involved in these two disease processes.

The treatment of rheumatologic disease in CVID is the same as for individuals who are not immune deficient, with the exception of the addition of adequate Ig replacement. However, when Ig therapy ameliorates or cures joint disease, an infectious etiology is more likely. Although hydroxychloroquine appears safe and modestly effective in rheumatoid disease, previous treatment with medications such as cyclophosphamide, azathioprine, and mycophenolate mofetil can reduce serum Ig, leading to some confusion about the underlying diagnosis [11].

\section{Granulomatous disease and autoimmunity}

Granulomatous lesions occur in the tissues of 5\% to 20\% of patients with CVID. These lesions are noncaseating and most prominent in the lung, liver, spleen, skin, bone marrow, and/or lymph nodes [12]. These lesions are commonly associated with significant lymphadenopathy and splenomegaly. Granulomatous disease in the lung may be confused with sarcoidosis prior to the recognition of hypogammaglobulinemia; this leads to delays in treatment of the immune defect and risk for severe infections [12,13]. Patients with known CVID may be found to have granulomata when a tissue is biopsied at a later time. However, the key features that distinguish the two diseases include reduced serum Ig with lack of antibody and history of recurrent infections in CVID. The etiology of granuloma remains unknown. For the most part, infectious agents have not been found. It has been tempting to suggest that the granuloma in CVID develops due to excess macrophage/monocyte activation in the face of defective B-cell and, often, T-cell defects, as these are commonly found [12]. For unclear reasons, more than half of CVID patients with granulomas also have had autoimmunity, usually ITP or AIHA $[12,13]$. Two of our patients with extensive pulmonary granulomas had life-threatening or fatal hemolytic anemia [6]. Genetic polymorphisms of the tumor necrosis factor (TNF) gene, TNF allele +488 , in individuals with granuloma, also have been described [14], with increased TNF- $\alpha$ in some [15]. From a diagnostic point of view, an increased serum angiotensin-converting enzyme can be found in CVID and other immune defects, and a positive Kveim test, characteristic of sarcoidosis, can also be positive in CVID [12].

Appropriate treatment of granulomatous infiltrations with or without autoimmunity in CVID presents a challenge, as immunosuppression is undesirable. However, when required, lowdose chronic oral steroids ( $10 \mathrm{mg} / \mathrm{d}$ or every other day) may be used. Hydroxychloroquine may be an additional treatment due to its inhibitory effects on macrophage/monocyte activation, antigen presentation, and TNF- $\alpha$ release [16]. TNF- $\alpha$ antagonists have been used with some success in cases of granulomatous diseases in CVID; however, they have not 
been of clear benefit in sarcoidosis. Thus, the utility of this therapy in CVID-associated granulomas remains undefined. Treatment of associated cytopenias would be as previously outlined.

\section{Inflammatory gastrointestinal disease}

Inflammatory bowel-like disease has been described in $6 \%$ to $10 \%$ of patients with CVID [5]. Whether this represents a form of autoimmunity or self-reactivity is difficult to clarify. A recent study of patients with CVID with concomitant gastrointestinal disease revealed a diverse histopathology, including intraepithelial lymphocytosis, lymphoid aggregates, granulomas, crypt distortion, and a characteristic lack of plasma cells. Histologic patterns in CVID biopsies often mimic diseases such as graft-versus-host disease, lymphoid hyperplasia, and villous atrophy resembling celiac sprue, although wheat avoidance is almost always unhelpful [17]. Liver disease with persistently elevated liver enzymes, including primary biliary cirrhosis and what appears to be autoimmune hepatitis, has also been observed [5]. In a survey of 10 CVID patients with elevated liver enzymes, biopsies demonstrated mild portal and mild to moderate lobular chronic inflammation, and plasma cells were absent in all specimens. Four patients showed small numbers of scattered portal and/or lobular granulomas, and three of the patients had a coexisting diagnosis of sarcoidosis [18]. Pernicious anemia is another autoimmune disease noted in a small percentage (1\% to $9 \%$ ) of almost all series of patients with CVID $[2 \cdot \bullet, 5]$.

Intestinal inflammation in CVID can be difficult to treat. Even in cases in which no organisms are found, antibiotics are often prescribed. Prolonged oral steroid treatment is not ideal given the risk of immunosuppression, although it is likely to reduce symptoms. Enteric-coated oral budesonide (Entocort EC; AstraZeneca, Wilmington, DE) is a potent locally acting corticosteroid that has been used in CVID-associated inflammatory bowel disease at doses of $3 \mathrm{mg}$ three times daily. Immunomodulators 6-mercaptopurine or azathioprine may be required and can be used in low doses $(1.5 \mathrm{mg} / \mathrm{kg}$ for 6 mercaptopurine, $2.5 \mathrm{mg} / \mathrm{kg}$ for azathioprine). TNF inhibitors (eg, infliximab) have also been used in a few cases.

\section{Immune Defects in CVID}

As the cardinal manifestation is hypogammaglobulinemia, CVID is generally classified as a B-cell defect, although as discussed subsequently, other cellular defects are known, suggesting that CVID is in many ways a form of combined defect. Peripheral B cells are usually normal in number but may be reduced or nearly absent; however, in all cases, B-cell maturation is abnormal, with variably deficient Ig production in vivo and a lack of plasma cells in tissues. Because an appropriate environment and specific signals are required to differentiate naïve $\mathrm{B}$ cells into antibody-secreting cells, many investigators over the years have studied in vitro Ig synthesis in CVID using a number of stimulators. These studies revealed that patients usually can be grouped according to functional losses, with some patients unable to produce any Ig in culture and others able to produce modest amounts. In response to Staphylococcus aureus Cowan and interleukin (IL)-2, for example, the B cells of some patients fail to produce any Ig. A second group produces only IgM, with little or no $\mathrm{IgG}$, and a third group produces IgM and IgG in normal or near-normal quantities [19]. B cells of some CVID patients produce Ig if stimulated by the polyclonal B-cell activator $\mathrm{CpG}$ oligonucleotide [20]; however, the resulting isotype is mostly IgM and less IgG or IgA. That B cells of about $40 \%$ of CVID patients, when cultured in vitro with anti-CD40 and IL-4, undergo some differentiation and synthesize $\operatorname{IgG}$ demonstrates the likelihood that patients have B lymphocytes capable of some antibody function, potentially permitting selfreactivity [21]. 
Similar to the hyper IgM syndrome, CVID B cells exhibit impaired somatic hypermutation [22] and a relative lack of $\mathrm{CD} 27^{+}$memory B cells. CD27, a member of the TNF receptor gene superfamily, is expressed on about $30 \%$ to $40 \%$ of B lymphocytes in the blood of adults and is used to distinguish memory B cells. Although there are modestly reduced numbers of memory B cells in peripheral blood in CVID, there are even greater losses of isotype switched $\left(\mathrm{IgD}^{-}, \mathrm{IgM}^{-}, \mathrm{CD} 27^{+}\right)$memory B cells [23]. Clinically, very low numbers of these isotype switched memory B cells is a useful means of classifying patients into biologically relevant groups $[23,24 \bullet \bullet]$. Loss of isotype switched memory B cells $(<0.4 \%)$ is correlated with lower serum IgG levels and poorer antibody responses [25,26]. Loss of these cells is also associated with the development of autoimmunity, lymphoid hyperplasia, splenomegaly, and granulomatous disease $[23,24 \bullet \bullet, 27]$, suggesting that defective class switch and loss of hypermutation in CVID may lead to an inability to exclude autoimmune clones.

Another potentially important factor in autoimmunity is B-cell-activating factor (BAFF), a cytokine produced by cells of myeloid origin that is important for the survival and maturation of B cells [28•]. Over-expression of BAFF in mice leads to B-cell hyperplasia, splenomegaly, and autoimmunity. A substantial body of literature demonstrates that BAFF (and a proliferation-inducing ligand [APRIL]) are present in excess amounts in the sera of patients with systemic autoimmune disease (eg, rheumatoid arthritis, systemic lupus erythematosus, and systemic sclerosis). Because some of the clinically problematic hallmarks of CVID are autoimmune disease, striking lymphadenopathy, and splenomegaly, levels of APRIL and BAFF were determined in the serum of patients. The results demonstrated that BAFF and APRIL were greatly elevated in most patients with CVID; however, these levels were not significantly related to autoimmunity or lymphoid hyperplasia present in some, suggesting that the role of these factors in CVID is not yet well understood [29•].

Significant and numerous abnormalities of T-cell function, cytokine secretion, cell adhesion, and dendritic cells also have been described in CVID. These include lymphopenia, poor lymphocyte proliferation, skewed $\mathrm{CD} 8^{+}$cell numbers, a lack of antigen-specific $\mathrm{T}$ cells, a restricted $\mathrm{T}$-cell receptor repertoire with oligoclonal expansion of $\mathrm{CD} 8^{+} \mathrm{T}$ cells, and decreased $\mathrm{CD}^{+}{ }^{+} \mathrm{CD} 45 \mathrm{RA}^{+} \mathrm{T}$ cells. CVID T lymphocytes produce markedly decreased amounts of IL-2, interferon- $\gamma$, IL-4, IL-5, and IL-10. Other T-cell defects include impaired T-cell activation following stimulation with anti-CD3, impaired intracellular tyrosine kinase expression following T-cell receptor ligation, reduced Zap-70 mobilization, deficient CD28 co-signaling, and accelerated T-cell death associated with increased expression of CD95. Although these significant but complex cellular defects may lead to a cytokine or germinal center environment that impairs removal of self-reactive $T$ cells, in most cases, these studies have not made specific associations with the development of autoimmunity in patients. However, several investigators have found that a reduced frequency of T-regulatory cells $\left(\mathrm{CD}^{+}, \mathrm{CD}^{2} 5^{+}\right.$, forkhead box $\left.\mathrm{P}^{+}\right)$was related to autoimmunity, splenomegaly, or other inflammatory markers in CVID [30,31]. The cellular defects potentially related to autoimmunity in CVID are summarized in Table 2.

\section{Genetics of CVID}

More than $90 \%$ of individuals with CVID do not have an immune-deficient family member, suggesting that this syndrome results from multigenic causes. In about $10 \%$ of families, one or more additional family members have CVID or, more often, selective IgA deficiency [32]. However, non-immune-deficient family members may have an increased incidence of autoimmunity. Linkage analysis in families in which both CVID and selective IgA deficiency occur has long implicated the HLA-DQ/DR haplotype as a susceptibility locus 
[33]. Recently, genetic variations in $M s h 5$, a gene involved in regulation of meiotic nonhomologous recombination potentially contributing to Ig class switch recombination, were noted in CVID patients [34].

However, several single gene lesions have been found to lead to the CVID phenotype: mutations of the T-cell activation marker the inducible co-stimulator in one extended kindred [35] and CD19 in a few families [36]. Disruption of BAFF receptor in mice results in an immunologic phenotype similar to CVID, and this receptor was found to have a significant mutation in one patient [37]. More complex are mutations in the gene encoding the related receptor known as transmembrane activator and calcium-modulating ligand interactor (TACI, TNFRSR13B), which is expressed mostly on mature B cells and found in $7 \%$ to $10 \%$ of patients $[38,39]$. Although mutations in TACI are clearly associated with CVID, some of the same mutations can be found in non-immunedeficient family members and a few normal controls $[40 \bullet, 41 \bullet]$. However, CVID patients with mutations in TACI have an increased incidence of autoimmunity. In a study of 176 patients, mutations in TACI were found in $13(7.3 \%)$, six of whom (46\%) had episodes of autoimmune thrombocytopenia, which was in contrast to the $12 \%$ of 163 patients without mutations. Splenomegaly and splenectomy were also significantly increased in the group with TACI mutations $(P=0.012$

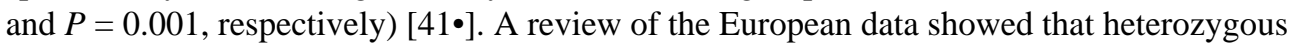
inheritance of the C104R mutation was also associated with autoimmunity and lymphoid hyperplasia in this cohort [42••]. As $\mathrm{TACI}^{-/-}$mice develop splenomegaly, lymphadenopathy, lymphoma, and a fatal autoimmune syndrome similar to human SLE, it seems probable that this receptor exerts selected inhibitory effects that are impaired in patients with CVID and mutations.

\section{Conclusions}

Primary immune defects commonly lead to autoimmune manifestations. These may be organ or tissue based. From the medical perspective, they are difficult to treat, as prolonged immune suppression, undesirable in these patients, may be required. The pathogenesis of autoimmunity in immune deficiency is unclear for the most part, but careful dissection of immune mechanisms in some has led to greater understanding of autoimmunity in general. The best examples of these include the mutations in CD95 in autoimmune lymphoproliferative disorder, which lead to defective apoptosis and autoimmune cytopenias, and mutations in the IL-2 receptor, signal transducer and activator transcription 5, and forkhead box P3, which lead to loss of functional T-regulatory cells. For other immune defects, including CVID, the pathogenesis of autoimmunity remains more obscure, although recent genetic studies provided some illumination. However, the heterogeneity in both pathogenesis and clinical complications in CVID makes these investigations challenging.

\section{Acknowledgments}

This work was supported by National Institutes of Health grants AI-101093, AI-467320, and AI-48693 and National Institute of Allergy and Infectious Diseases contract 03-22 (to Dr. Cunningham-Rundles).

\section{References and Recommended Reading}

Papers of particular interest, published recently, have been highlighted as:

- Of importance

•• Of major importance 
1. Geha RS, Notarangelo LD, Casanova JL, et al. Primary immunodeficiency diseases: an update from the International Union of Immunological Societies Primary Immunodeficiency Diseases Classification Committee. J Allergy Clin Immunol 2007;120:776-794. [PubMed: 17952897]

2••. Chapel H, Lucas M, Lee M, et al. Common variable immunodeficiency disorders: division into distinct clinical phenotypes. Blood 2008;112:277-286. This study describes the clinical phenotypes of 334 patients in the European CVID registry to define distinct clinical phenotypes. [PubMed: 18319398]

3. Cunningham-Rundles C. Common variable immunodeficiency. Curr Allergy Asthma Rep 2001;1:421-429. [PubMed: 11892068]

4. Quinti I, Soresina A, Spadaro G, et al. Long-term follow-up and outcome of a large cohort of patients with common variable immunodeficiency. J Clin Immunol 2007;27:308-316. [PubMed: 17510807]

5. Cunningham-Rundles C, Bodian C. Common variable immunodeficiency: clinical and immunological features of 248 patients. Clin Immunol 1999;92:34-48. [PubMed: 10413651]

6. Wang J, Cunningham-Rundles C. Treatment and outcome of autoimmune hematologic disease in common variable immunodeficiency (CVID). J Autoimmun 2005;25:57-62. [PubMed: 15994061]

7. Michel M, Chanet V, Galicier L, et al. Autoimmune thrombocytopenic purpura and common variable immunodeficiency: analysis of 21 cases and review of the literature. Medicine (Baltimore) 2004;83:254-263. [PubMed: 15232313]

8. Swierkot J, Lewandowicz-Uszynska A, Chelbicki A, et al. Rheumatoid arthritis in a patient with common variable immunodeficiency: difficulty in diagnosis and therapy. Clin Rheumatol 2006;25:92-94. [PubMed: 15940551]

9. Bloom KA, Chung D, Cunningham-Rundles C. Osteoarticular infectious complications in patients with primary immunodeficiencies. Curr Opin Rheumatol 2008;20:480-485. [PubMed: 18525364]

10. Fernandez-Castro M, Mellor-Pita S, Citores MJ, et al. Common variable immunodeficiency in systemic lupus erythematosus. Semin Arthritis Rheum 2007;36:238-245. [PubMed: 17276173]

11. Yong PF, Aslam L, Karim MY, et al. Management of hypogammaglobulinaemia occurring in patients with systemic lupus erythematosus. Rheumatology (Oxford) 2008;47:1400-1405. [PubMed: 18625660]

12. Mechanic LJ, Dikman S, Cunningham-Rundles C. Granulomatous disease in common variable immunodeficiency. Ann Intern Med 1997;127:613-617. [PubMed: 9341059]

13. Morimoto Y, Routes JM. Granulomatous disease in common variable immunodeficiency. Curr Allergy Asthma Rep 2005;5:370-375. [PubMed: 16091208]

14. Mullighan CG, Fanning GC, Chapel HM, et al. TNF and lymphotoxin-alpha polymorphisms associated with common variable immunodeficiency: role in the pathogenesis of granulomatous disease. J Immunol 1997;159:6236-6241. [PubMed: 9550427]

15. Aukrust P, Lien E, Kristoffersen AK, et al. Persistent activation of the tumor necrosis factor system in a subgroup of patients with common variable immunodeficiency-possible immunologic and clinical consequences. Blood 1996;87:674-681. [PubMed: 8555490]

16. Kalia S, Dutz JP. New concepts in antimalarial use and mode of action in dermatology. Dermatol Ther 2007;20:160-174. [PubMed: 17970883]

17. Daniels JA, Lederman HM, Maitra A, et al. Gastrointestinal tract pathology in patients with common variable immunodeficiency (CVID): a clinicopathologic study and review. Am J Surg Pathol 2007;31:1800-1812. [PubMed: 18043034]

18. Daniels JA, Torbenson M, Vivekanandan P, et al. Hepatitis in common variable immunodeficiency. Hum Pathol 2009;40:484-488. [PubMed: 19084266]

19. Bryant A, Calver NC, Toubi E, et al. Classification of patients with common variable immunodeficiency by B cell secretion of $\operatorname{IgM}$ and $\mathrm{IgG}$ in response to anti-IgM and inter-leukin-2. Clin Immunol Immunopathol 1990;56:239-248. [PubMed: 2165880]

20. Cunningham-Rundles C, Radigan L, Knight AK, et al. TLR9 activation is defective in common variable immune deficiency. J Immunol 2006;176:1978-1987. [PubMed: 16424230]

21. Eisenstein EM, Chua K, Strober W, et al. B cell differentiation defects in common variable immunodeficiency are ameliorated after stimulation with anti-CD40 antibody and IL-10. J Immunol 1994;152:5957-5968. [PubMed: 7515919] 
22. Bonhomme D, Hammarstrom L, Webster D, et al. Impaired antibody affinity maturation process characterizes a subset of patients with common variable immunodeficiency. J Immunol 2000;165:4725-4730. [PubMed: 11035117]

23. Warnatz K, Denz A, Drager R, et al. Severe deficiency of switched memory B cells $(\mathrm{CD} 27(+) \operatorname{IgM}(-) \operatorname{IgD}(-))$ in subgroups of patients with common variable immunodeficiency: a new approach to classify a heterogeneous disease. Blood 2002;99:1544-1551. [PubMed: 11861266]

24••. Wehr C, Kivioja T, Schmitt C, et al. The EUROclass trial: defining subgroups in common variable immunodeficiency. Blood 2008;111:77-85. This was a European multicenter trial of 303 patients with CVID to develop a classification scheme based on flow cytometric B-cell phenotyping and clinical course, including granulomatous disease, autoimmune cytopenia, and splenomegaly. [PubMed: 17898316]

25. Alachkar H, Taubenheim N, Haeney MR, et al. Memory switched B cell percentage and not serum immunoglobulin concentration is associated with clinical complications in children and adults with specific antibody deficiency and common variable immunodeficiency. Clin Immunol 2006;120:310-318. [PubMed: 16782407]

26. Ko J, Radigan L, Cunningham-Rundles C. Immune competence and switched memory B cells in common variable immunodeficiency. Clin Immunol 2005;116:37-41. [PubMed: 15925830]

27. Sanchez-Ramon S, Radigan L, Yu JE, et al. Memory B cells in common variable immunodeficiency: clinical associations and sex differences. Clin Immunol 2008;128:314-321. [PubMed: 18620909]

28•. Mackay F, Silveira PA, Brink R. B cells and the BAFF/APRIL axis: fast-forward on autoimmunity and signaling. Curr Opin Immunol 2007;19:327-336. This was a comprehensive review of BAFF/APRIL signaling related to autoimmunity. [PubMed: 17433868]

29• . Knight AK, Radigan L, Marron T, et al. High serum levels of BAFF, APRIL, and TACI in common variable immunodeficiency. Clin Immunol 2007;124:182-189. This study quantified levels of TACI ligands APRIL, BAFF, and TACI in 77 patients with CVID. Increased serum levels of BAFF, APRIL, and TACI were detected; however, there was no correlation between levels and autoimmunity, lymphadenopathy, splenomegaly, B-cell numbers, or mutations in TACI. [PubMed: 17556024]

30. Genre J, Errante PR, Kokron CM, et al. Reduced frequency of CD4(+)CD25(HIGH)FOXP3(+) cells and diminished FOXP3 expression in patients with common variable immunodeficiency: a link to autoimmunity? Clin Immunol. 2009 Apr 23; (Epub ahead of print).

31. Yu GP, Chiang D, Song SJ, et al. Regulatory T cell dysfunction in subjects with common variable immunodeficiency complicated by autoimmune disease. Clin Immunol 2009;131:240-253. [PubMed: 19162554]

32. Vorechovsky I, Zetterquist H, Paganelli R, et al. Family and linkage study of selective IgA deficiency and common variable immunodeficiency. Clin Immunol Immunopathol 1995;77:185192. [PubMed: 7586726]

33. Schaffer AA, Salzer U, Hammarstrom L, et al. Decon-structing common variable immunodeficiency by genetic analysis. Curr Opin Genet Dev 2007;17:201-212. [PubMed: 17467261]

34. Sekine H, Ferreira RC, Pan-Hammarstrom Q, et al. Role for Msh5 in the regulation of Ig class switch recombination. Proc Natl Acad Sci U S A 2007;104:7193-7198. [PubMed: 17409188]

35. Grimbacher B, Hutloff A, Schlesier M, et al. Homozygous loss of ICOS is associated with adultonset common variable immunodeficiency. Nat Immunol 2003;4:261-268. [PubMed: 12577056]

36. van Zelm MC, Reisli I, van der Burg M, et al. An antibody-deficiency syndrome due to mutations in the CD19 gene. N Engl J Med 2006;354:1901-1912. [PubMed: 16672701]

37. Goldacker S, Warnatz K. Tackling the heterogeneity of CVID. Curr Opin Allergy Clin Immunol 2005;5:504-509. [PubMed: 16264329]

38. Castigli E, Wilson SA, Garibyan L, et al. TACI is mutant in common variable immunodeficiency and IgA deficiency. Nat Genet 2005;37:829-834. [PubMed: 16007086] 
39. Salzer U, Chapel HM, Webster AD, et al. Mutations in TNFRSF13B encoding TACI are associated with common variable immunodeficiency in humans. Nat Genet 2005;37:820-828. [PubMed: 16007087]

40•. Pan-Hammarstrom Q, Salzer U, Du L, et al. Reexamining the role of TACI coding variants in common variable immunodeficiency and selective IgA deficiency. Nat Genet 2007;39:429-430. This was a study analyzing TNFRSF13B in 115 Swedish, 154 German, and 155 US individuals with CVID. The study demonstrated a significant increase in the frequency of the C104R, A181E, and ins204A sequence variants in TNFRSF13B, suggesting their involvement in the development of CVID. [PubMed: 17392797]

41 . Zhang L, Radigan L, Salzer U, et al. Transmembrane activator and calcium-modulating cyclophilin ligand interactor mutations in common variable immunodeficiency: clinical and immunologic outcomes in heterozygotes. J Allergy Clin Immunol 2007;120:1178-1185. In this study, TACI was sequenced from DNA of 176 individuals with CVID and their family members. Mutations in TACI were found to predispose CVID patients to autoimmunity and lymphoid hyperplasia; however, non-immune-deficient family members had similar mutations, suggesting that additional genetic or environmental factors are required to induce immune deficiency. [PubMed: 17983875]

42••. Salzer U, Bacchelli C, Buckridge S, et al. Relevance of biallelic versus monoallelic TNFRSF13B mutations in distinguishing disease-causing from risk-increasing TNFRSF13B variants in antibody deficiency syndromes. Blood 2009;113:1967-1976. In this study of 50 individuals with TNFRSF 13B alterations, 13 new sequence variants were found. However, the only significant association of a specific TACI variant with CVID was that of TACI C104R. Heterozygosity for $\mathrm{C} 104 \mathrm{R}$ was found to be associated with low numbers of $\mathrm{IgD}^{-} \mathrm{CD} 27^{+} \mathrm{B}$ cells, benign lymphoproliferation, and autoimmune complications, suggesting that C104R heterozygosity increases the risk for CVID-associated disorders. [PubMed: 18981294]

43. Warnatz K, Wehr C, Drager R, et al. Expansion of CD19(hi)CD21(lo/neg) B cells in common variable immunodeficiency (CVID) patients with autoimmune cytopenia. Immunobiology 2002;206:502-513. [PubMed: 12607725]

44. Yong PF, Workman S, Wahid F, et al. Selective deficits in blood dendritic cell subsets in common variable immunodeficiency and X-linked agammaglobulinaemia but not specific polysaccharide antibody deficiency. Clin Immunol 2008;127:34-42. [PubMed: 18295543]

45. Giovannetti A, Pierdominici M, Mazzetta F, et al. Unravelling the complexity of T cell abnormalities in common variable immunodeficiency. J Immunol 2007;178:3932-3943. [PubMed: 17339494]

46. Cunningham-Rundles C, Radigan L. Deficient IL-12 and dendritic cell function in common variable immune deficiency. Clin Immunol 2005;115:147-153. [PubMed: 15885637]

47. Sneller MC. Common variable immunodeficiency. Am J Med Sci 2001;321:42-48. [PubMed: 11202479]

48. Holm AM, Aukrust P, Damas JK, et al. Abnormal inter-leukin-7 function in common variable immunodeficiency. Blood 2005;105:2887-2890. [PubMed: 15598813]

49. Rezaei N, Amirzagar AA, Shakiba Y, et al. Proinflammatory cytokine gene single nucleotide polymorphisms in common variable immunodeficiency. Clin Exp Immunol 2009;155:21-27. [PubMed: 19076825]

50. Eisenstein EM, Jaffe JS, Strober W. Reduced interleukin-2 (IL-2) production in common variable immunodeficiency is due to a primary abnormality of CD4+ T cell differentiation. J Clin Immunol 1993;13:247-258. [PubMed: 7901231] 
Table 1

Autoimmune diseases associated with common variable immunodeficiency

Dermatologic
Alopecia totalis
Vitiligo
Psoriasis
Hematologic
Immune thrombocytopenic purpura
Autoimmune hemolytic anemia
Autoimmune neutropenia
Endocrinologic
Hyperthyroidism
Hypothyroidism
Rheumatologic
Vasculitis
Systemic lupus erythematosus
Rheumatoid arthritis
Juvenile rheumatoid arthritis
Sicca syndrome
Arimartrointestinal
Autoimmatory bowel disease
Pernicious anemia
Atrophic gastritis


Table 2

Pathogenesis of autoimmunity

\begin{tabular}{|lc|}
\hline Defects & Studies \\
\hline B-cell defects & {$[22]$} \\
Impaired somatic hypermutation & {$[23,43]$} \\
Reduced isotype switched memory B cells & {$[44]$} \\
Increased numbers of CD21 ${ }^{-}$B cells & \\
T-cell defects & {$[30,31]$} \\
Reduced T-regulatory cells & {$[45]$} \\
Reduced CD4 and increased CD8 ${ }^{+}$T cells & \\
Dendritic defects & {$[46]$} \\
Reduced IL-12 production & {$[44,46]$} \\
Impaired differentiation, maturation, and function of dendritic cells & {$[38,39,40 \bullet, 41 \bullet, 42 \bullet \bullet$} \\
Genetics & \\
Mutations in TACI & {$[47-50]$} \\
Cytokines, growth factors & \\
Cytokine defects: IL-2, IL-4, IL-6, IL-7, IL-10, interferon- $\gamma$ & \\
Excess BAFF, APRIL & {$[29 \cdot]$} \\
\hline
\end{tabular}

APRIL — a proliferation-inducing ligand; BAFF-B-cell-activating factor; IL_-interleukin; TACI-transmembrane activator and calciummodulating ligand interactor. 